\title{
Application of Robust Optimization in Asset Portfolio
}

\author{
Qilin Li, Chuanliang Jia \\ School of Management Science and Engineering, Central University of Finance and Economics, \\ Haidian District, Beijing, China \\ cdliqilin@163.com,cnbjjcl@163.com
}

Keywords: finance; robust optimization; uncertainty; portfolio

\begin{abstract}
A portfolio robust optimization model is established concerning the uncertainties of future economic factors based on the domestic actual situation.Researches are carried out on the investment decision of fund companies, fund allocations of bankcard network.The optimal portfolio has been realized when the objective function and constraints are adjusted and improved according to every specific problem and the uncertain economic factors predicted using the uncertain set method.Then,the portfolio decision with both the feasible and the optimal is realized by means of robust optimization
\end{abstract}

\section{Introduction}

The uncertainty and risk investment income is a very important issue in the investment community. The major problem the investors facing is to carry on the effective configuration of the assets while balancing the maximization of the return on assets and the minimization of the risk under uncertain circumstance. This is the portfolio choice. In 1952, Markowitz quantified the risk of returns on equity with variance and puts forward the portfolio selection of mean - variance analysis method. This has opened the prologue of modern finance. More than 50 years, with the lead of Markowitz pioneering work, portfolio theory and practice research have made great progress. The mean-variance (MV) model, VaR model and all kinds of stochastic programming model have been widely used in the real life. However, a growing number of studies find that the mean -variance model and VaR model have the sensitive defects of input parameters (such as the expected return) error (D.Goldfaxb, 2003). This makes the optimal decision is usually not feasible. While all kinds of stochastic programming model take the parameter uncertainty problem into consideration, they require the known probability distribution of random parameters. The size of the problem and the number of scenarios that describe the uncertainty are exponential growth. This leads to form a very large scale optimization problem (Lu. Z, 2011). After the 1990s, as financial market uncertainties are increased and significant progress in the field of robust optimization is made, many scholars start to use the robust optimization method to consider uncertainty of portfolio and optimization problem as a whole. So the problem the classical model is sensitive to the input parameter errors can be solved, and the optimal portfolio selection under uncertainty is feasible.

Mulvey used the robust optimization model to study portfolio decision-making problem (Mulvey J M, 1995). Later, Ben-Tal proved that when the robust optimization is used to solve portfolio problems, the complexity of the model and the time needed for solving are the same as the classic model (Ben-Tal, 1999). But the results are more smoothly to decision results and more insensitive 
to input parameters. Then foreign scholars attaches great importance to the robust optimization problems on the portfolio, and combine with the UK, Italy, Brazil, the United States, Japan and other countries of the securities market or institutions such as Banks, insurance companies, fund management company to do the further research. They achieved rich results. But the uncertainty description method, these constraints and objective functions in the study do not perfectly match with the actual finance situation of our country (Fabozzi J F, 2007). According to the actual financial situation in our country and to the specific problem, we use the robust optimization model in Asset Portfolio. And the model of objective function and constraint condition was improved and adjusted. Considering the uncertainty of future economic factors, we research the investment of the fund company and capital allocation decisions of bank card network.

\section{The robust optimization model in portfolios}

\subsection{The Review of Classical Robust Optimization Theory}

The Robust optimization is being widely applied in various fields such as economic management and natural science, which has become an effective method to deal with the problem of uncertainty and has aroused great concern (Huang Xiaoyuan, 2007). The disadvantage of traditional optimization methods is that: they appear powerless and the optimization of the results often deviates from the actual situation when the internal parameters change as a result of external disturbance. Instead the robust optimization method is a good way to solve the optimization problem of uncertain conditions (Black F, 1992). Robust optimization will directly incorporate the uncertainty involved in the decision-making process into the optimization model, so that the solution of the problem is guaranteed to be feasible and optimal in all possible situations. So far, the robust optimization has progressed to the classical system of the current robust optimization theory, and the trend is still changing and developing.

The important thought of robust optimization is given in the referred paper (Mulvey, 1995). First, to describe the uncertainty, we need to introduce an uncertain set $D=\{1,2, \ldots S\}$. By defining the uncertain set, we can give a quantitative description to uncertainty and a variety of possibilities. We can get such quantitative description through evaluating the internal and external situation of the system, summarizing the historical data, and predicting the future events. But this description is different from conventional predictions and can include a collection of point estimation, such as point estimate of the confidence interval around or a set of scenarios. Second, we shall define Solution Robust and Model Robust. For uncertain concentration of each scene, if the model of the optimal solution can keep or close to the optimal, so we call them robust solution. If the optimal solution is always feasible, it is considered to be robust model (Zhu Shushang, 2004). The tradeoff between the robustness and the robustness of the model can be effectively weighed against the optimal and feasible questions. This special relationship ensures that there is no over-sensitivity to specific uncertainties, which is the fundamental goal of robust optimization.

\subsection{The Basic Framework of Robust Optimization Model of the Asset Portfolio}

We can summarize the basic framework of robust optimization model of the asset portfolio, which is described below (Wang Yuanying, 2007). The following is a classic portfolio selection model:

$$
\begin{gathered}
\max _{w} \mu^{T} W-\lambda W^{T} V W \\
\text { s.t } \quad W^{T} I=1
\end{gathered}
$$


$\mu$ is the n-dimensional column vector of the expected return of the asset; $w$ is the n-dimensional column vector of the asset portfolio weight; $\lambda$ is the risk aversion factor; $v$ is the covariance matrix; $I$ is the unit vector.

It is obvious that the optimal asset combination can be obtained precisely based on formula (1) if the input parameters of the expected income vector and covariance matrix can be predicted accurately. However, the expected returns in reality are unpredictable and can sometimes be biased. Small changes in expected returns can lead to big changes in asset portfolio allocation. As a result, the general situation is that people are optimized based on inaccurate estimates. Considering these estimates error, an uncertain set closed to $\hat{\mu}$ estimation is required. And we solve the optimization of all vectors in the uncertainty set. An indefinite set can be as follows:

$$
U_{\delta}(\hat{\mu})=\left\{\mu\left|\mu_{i}-\hat{\mu}_{i} \leq \delta_{i}\right|, i=1, \ldots, n\right\}
$$

The uncertainty set $U_{\delta}(\hat{\mu})$ includes all of the vectors $\mu=\left(\mu_{1}, \ldots, \mu_{n}\right)$.Each $\mu_{i}$ belongs to $\left[\hat{\mu}_{i}-\delta_{i}, \hat{\mu}_{i}+\delta_{i}\right]$.

Then, to solve the optimal problem, the goal is to realize that even if the value in the real world is the worst value in the uncertainty concentration, the asset portfolio allocation is still optimal. That is:

$$
\max _{W}\left\{\min _{\mu \in J_{\delta}(\hat{\mu})}\left(\mu^{T} W\right)-\lambda W^{T} V W\right\}
$$

To facilitate calculation, formula (3) is rewritten as:

$$
\max _{W} \hat{\mu}^{T} W-\delta^{T}|W|-\lambda W^{T} V W
$$

$|W|$ is the absolute value of the weight vector. Further, we're going to introduce n dimensional vectors $\psi$ to replace $|W|$.Then:

$$
\begin{gathered}
\max _{W \cdot \psi} \hat{\mu}^{T} W-\delta^{T} \psi-\lambda W^{T} V W \\
\quad \text { s.t } W^{T} I=1 \\
\psi_{i} \geq w_{i} ; \psi_{i} \geq-w_{i}, i=1,2, \ldots N
\end{gathered}
$$

This framework can achieve the robustness of the asset portfolio in two ways. One is that the optimization result reduces the weight of the assets with the larger estimate error. Secondly, the objective function $\psi$ is the risk preference for estimation error penalty. Its size is correlated with $\delta$ and can be controlled by the size and structure of the uncertainty set.

\subsection{The Objective Function of Robust Optimization Model of The Asset Portfolio}

There are two kinds of objective functions in the basic framework of robust optimization model of the asset portfolio.

(1)The classical mean -variance function.

$$
f_{1}=\max \alpha
$$


$\alpha$ is the upper bound variable which is introduced deviation fluctuation. Its practical significance is to solve the optimal asset portfolio in the worst case. The purpose of this method is to solve the problem of estimation error of input parameters by solving the optimal solution in the worst-case scenario. Under this kind of objective function, the robust quadratic programming and linear matrix inequality method are usually used to solve the problem. To transform a mean-variance function into a robust quadratic programming with linear matrix inequality (IMI) problem has three advantages. Firstly we own effective solution tool at present and using MATLAB to solve related problems are very practical and very convenient with high maneuverability; Secondly allows for the balance and synthesis of many different, even contradictory constraints and requirements in the optimization process; Lastly it is particularly suitable for parameter uncertainties.

(2) Expand the expected utility function

$$
f_{2}=\min \sum_{S \in \Omega} \sigma\left(x, y_{s}\right)+\omega \sum_{S \in \Omega} p_{s} e_{s}^{T} e_{s}
$$

$x$ represents design variables whose optimal value is independent of the uncertain parameters. Once an uncertain parameter is observed, the control variable $y_{s}$ can be adjusted. The optimal value of the control variable depends on the implementation of uncertain parameters, and the value of the design variable. $e_{s}(\forall s \in \Omega)$ is the deviation vector corresponding to each scene in the uncertain set $\Omega$, which reflects the unfeasibility of the solution in the situation $s$.The first item of the objective function represents the robustness of the solution, which can be expressed in terms of the least cost or maximum benefit function, and can be used in other forms according to the characteristics of the problem and the preference of the decision maker. The second item of the objective function represents the robustness of the model, and $\omega$ reflects the weights of the robustness of the model. The larger the value is, the greater the penalty for deviation error is, and the stronger the robustness of the model is. Conversely, the weaker the robustness of the model is.

\section{Application of robust optimization model in asset portfolio}

\subsection{Application in Investment Fund Management}

The investment fund is managed by the fund manager and the use of funds. Through the portfolio investment of financial instruments such as stock, they can reduce risk and make profit for investors. Therefore, the important problem that an investment fund manager must face is to determine the weight of the selected stocks (William t. zimba, 2003). Given the characteristics of the investment fund, the formula (11)-(13) is given under the framework of robust optimization model of the asset portfolio. The objective function (9) is adjusted as formula (11), and the target is the stock portfolio of the target income constraint and the minimum tracking error under the worstcase scenario.

$$
\begin{gathered}
\min \alpha \\
\text { s.t. }\left[\begin{array}{cc}
\alpha & \left(\omega-\omega_{B}\right)^{T} V_{k} \\
V_{k}\left(\omega-\omega_{B}\right) & V_{k}
\end{array}\right] \geq 0 \\
\left(\omega-\omega_{B}\right)^{T}\left(\mu_{k}-r I\right) \geq R_{k}, k=1,2, \ldots, m
\end{gathered}
$$

The model has three certain parameters $\omega_{B}, R_{k}$ and $r . \omega_{B}$ is the weight of benchmark asset portfolio. The benchmark asset portfolio is the asset portfolio based on the predetermined target. $R_{k}$ 
represents $k$ target earnings. $R$ is the risk-free rate; The model has two subscript variables $i$ and $k$, $i$ represents various securities, $i=1,2, \ldots, n$. $k$ represents expected return, covariance matrix and the number of target profit. The change of economic environment of investment cycle will lead to different expected return and covariance, and therefore we use the $k$ expected return and covariance under restriction of $k$ target gains to represent the sets of uncertainty of the future market change, $k=1,2, \ldots m$.

According to $\alpha-\left(\omega-\omega_{B}\right)^{T} V_{k}\left(\omega-\omega_{B}\right) \geq 0$, the constraint conditions (12) are obtained by the transformation of Sehur. Formula (13) is the expected income constraint of asset portfolio. Other constraint conditions are formula (7)-(8).

The "southern consumption and processing" fund is an open-ended fund owned by the southern fund management company, which has basically the same management style as some funds in China. They adjust the proportion of investment every quarter. The stock set is constructed according to the basic analysis method. After the stock set is constructed, the specific investment weight will be selected under the restricted single income target constraint.

The uncertainty set in the model consists of two expected income vectors and two covariance matrices. The calculation method is shown in the referred paper (Gao Ying, 2009). We shall calculate by using LMI toolbox of Matlab software, and get the stock set according to the 10 stocks selected by "southern consumption and processing" fund every quarter. Then we obtained a total of eight quarters of robust weighting stock option of "southern consumption and progressive" fund in 2015-2016, and then get the corresponding investment yields respectively.. The results compared with the actual fund performance are shown in table 1 and figure 2 .

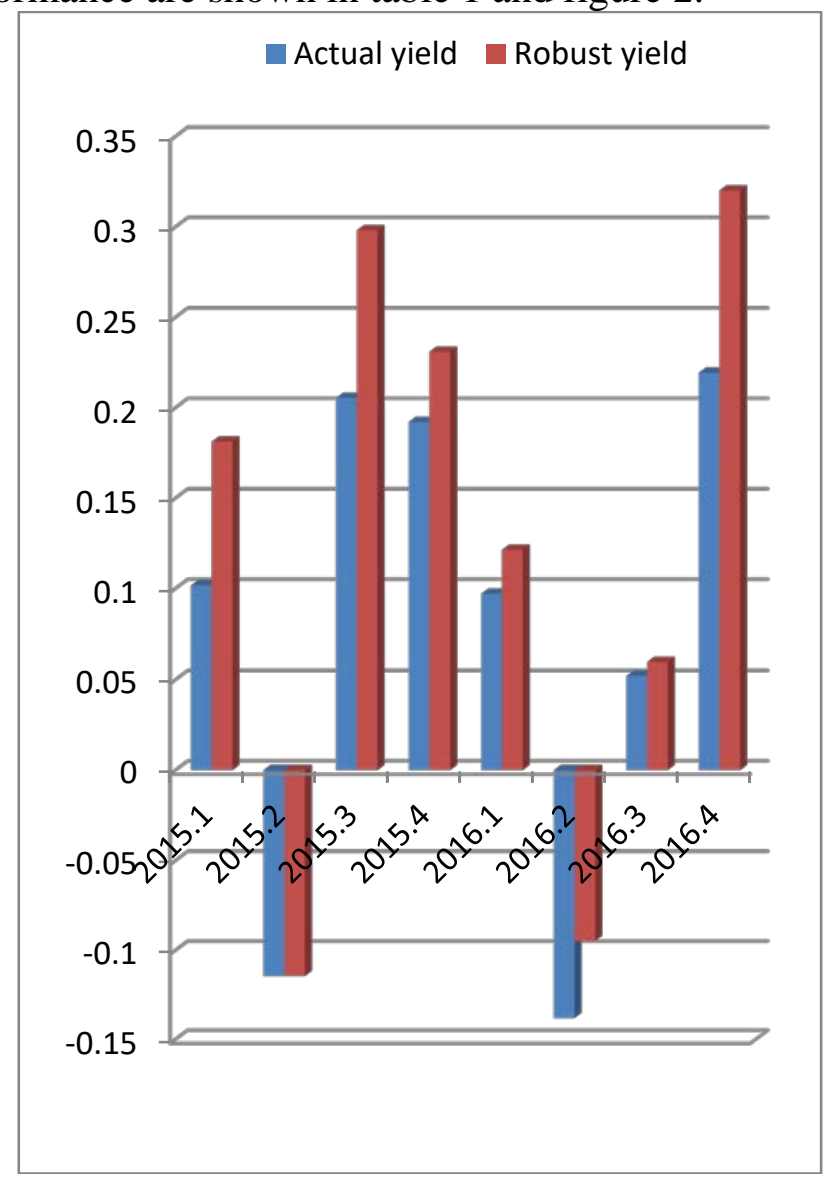

Figure 1 Comparison of the "southern consumption and processing" robust quarterly yield and actual quarterly yield 
Table 1 Comparison of "southern consumption and processing" robust quarterly yield and actual quarterly yield

\begin{tabular}{|l|l|l|l|l|l|l|l|l|}
\hline Time & 15.1 & 15.2 & 15.3 & 15.4 & 16.1 & 16.2 & 16.3 & 16.4 \\
\hline Actual yield & 0.10 & -0.11 & 0.20 & 0.19 & 0.09 & -0.13 & 0.05 & 0.21 \\
\hline Robust yield & 0.18 & -0.11 & 0.29 & 0.23 & 0.12 & -0.09 & 0.05 & 0.32 \\
\hline
\end{tabular}

\subsection{Aapplication of Fund Allocation of Bank Card Network}

The all-weather and all-day operation of the card network requires the banks to meet the capital requirements of the cardholder at any time. Of course, from the perspective of bank management, the fund preparation of the network should be fully utilized and should not be overused. However, in actual operation card network, stock market prices, interest rates, the existence of uncertain factors such as price, can cause the uncertainty of cardholder demand (Wang Yuanying, 2007). This will bring a contradiction between the normal system operation and minimum operation network cost. As a result, in the uncertain situation, it is an urgent problem for the card network to ensure the network all-weather operation and realize the minimization of system operation cost.

There are $n$ banks in the bank card network. The definition $i$ represents the $i$ - th bank of the network $(i=1,2, \ldots n) . j$ means the $j$-th bank of the network $(j=1,2, \ldots n)$. Other parameters: $f_{i}$ is the fixed entry fee for bank $i h_{i}$ and $g_{i}$ is respectively the unit cost of establishing cash and non-cash payment of network for bank $i . v_{i}$ and $u_{i}$ are respectively the unit variable cost for the bank $i$ to meet the cash and non-cash demand; $n$ is the number of banks in the network; $\omega$ is the robust weight of this model, which represents the disgust degree of the bias between prepared capital and real demand; $p_{s}$ is the probability of occurrence in the $s$ - $t$ h scenario $s \in \Omega=\{1,2,3, \ldots, s\}, \sum_{s \in \Omega} p_{s}=1$. $x_{i}$ and $y_{i}$ are the cash and non-cash preparation for bank $i$.Accordingly the cash and non-cash preparation for scenario $s$ are respectively expressed by $x_{i}^{\prime}$ and $y_{i}^{\prime} . a_{i}$ and $b_{i}$ are respectively the ability to handle cash and non-cash demand for bank $i$, which are also respectively the amount of cash and non-cash amount that bank $i$ can allocate in UnionPay network. $e_{1 i}^{s}$ is the deviation between cash preparation and real cash demand of bank $i$ and $e_{2 i}^{s}$ is the deviation between non-cash preparation and real non-cash demand for scenario $s$.

To minimum cost of UnionPay network, the objective function is obtained by equation (9)

$$
\min z=\sum_{i=1}^{n}\left(f_{i}+a_{i} h_{i}+b_{i} g_{i}\right)+\sum_{s \in \Omega} p_{s} \sum_{i=1}^{n}\left(x_{i}^{s} v_{i}+y_{i}^{s}\right)+\omega \sum_{s \in \Omega} p_{s} \sum_{i=1}^{n}\left[\left(e_{1 i}^{s}\right)^{2}+\left(e_{2 i}^{s}\right)^{2}\right]
$$

We choose some banks of China UnionPay network: Industrial and Commercial Bank of China, Agricultural Bank of China, Bank of Communication, China Construction Bank, China Merchants Bank and Bank of China. In 2016, the total amount of bank card transactions (consumption, transfer, deposit and withdrawal) of the six banks accounted for more than $90 \%$ of all the card issuing institutions in China, which is highly representative. The adjustment of the constraint conditions and the formation of the undefined set are shown in the referred paper (Gao Ying, 2007). We can use the general nonlinear optimization function (fmincon) with constraint in toolbox of Matlab7.01. We can get robust optimal solutions to uncertain money demand. Comparing with the optimal solution of certain demand (the average demand), we can find the results as shown in table 2 and table 3 .

Table 2 The robust solutions of fund allocation under uncertain capital requirements Unit: RMB 
100 million

\begin{tabular}{|l|l|l|l|l|}
\hline Banks & $x_{i}$ & $y_{i}$ & $a_{i}$ & $b_{i}$ \\
\hline 1 & 2405.665 & 0.000 & 2585.038 & 396.810 \\
\hline 2 & 4301.685 & 0.328 & 4374.069 & 213.499 \\
\hline 3 & 1186.626 & 173.867 & 1502.087 & 653.605 \\
\hline 4 & 2604.048 & 704.252 & 2680.009 & 770.924 \\
\hline 5 & 338.885 & 663.583 & 380.345 & 766.138 \\
\hline 6 & 780.260 & 1833.444 & 785.445 & 2108.39 \\
\hline $\begin{array}{l}\text { The value of } \\
\text { objective function }\end{array}$ & 2781.137 & & $\begin{array}{l}\text { Total cost } \\
\text { standard } \\
\text { deviation }\end{array}$ & $\begin{array}{l}6.1 \times 10^{-8} \\
(100 \mathrm{M})^{2}\end{array}$ \\
\hline
\end{tabular}

Table 3 Fund operation strategy under certain capital requirements Unit: RMB 100 million

\begin{tabular}{|l|l|l|l|l|}
\hline Banks & $x_{i}$ & $y_{i}$ & $a_{i}$ & $b_{i}$ \\
\hline 1 & 2419.105 & -93.049 & 2585.038 & 396.810 \\
\hline 2 & 4338.395 & -47.938 & 4374.069 & 213.499 \\
\hline 3 & 1199.109 & 184.398 & 1502.087 & 653.605 \\
\hline 4 & 2617.190 & 704.252 & 2680.009 & 770.924 \\
\hline 5 & 351.892 & 693.938 & 380.345 & 766.138 \\
\hline 6 & 793.392 & 1838.226 & 785.445 & 2108.39 \\
\hline $\begin{array}{l}\text { The value of } \\
\text { objective }\end{array}$ & 2502.09 & $\begin{array}{l}\text { Total cost } \\
\text { standard } \\
\text { deviation }\end{array}$ & $\begin{array}{l}9.3 \times 10^{-8} \\
(100 \mathrm{M})^{2}\end{array}$ \\
\hline
\end{tabular}

\section{Conclusions}

The robust optimization model is introduced in this paper, and the application of the robust optimization model is presented. Considering the actual situation in China, the paper studies the problem of investment fund decision making and the allocation fund of bank card network. We can get the following conclusions:

(1) The application analysis of investment fund. It can be seen from the table and graph that: the weight of the portfolio is different. Because robust optimization is characterized by two expected returns and two covariance matrices. The model established in this paper considers the possibility that the stock will change in price in the future, so the selection of its weight will be more in line with the interests of investors. The optimal investment income of the robust optimization model is higher than the real income. In other words, the framework of robust optimization model of asset portfolio can be used in the real market of our country.

(2) The analysis of the allocation fund of bankcard network. It can be seen from the table and graph that: the optimal strategy made by the robust optimization model increases by $11.2 \%$, compared with the actual situation. This indicates that the robust optimization results are relatively conservative. The total cost standard deviation of robust solution is 36.9 percent lower than the optimal strategy under certain requirements. This shows that robust optimization can ensure the stable bank savings and the smooth operation of the bank's funds. It also makes the bank own better anti-disturbance ability. The characteristic of the robust optimization is that the better stability can be obtained with a slightly higher cost. 


\section{References}

[1] Ben-Tal. A, Nemirovski. A,Robust solutions of uncertain linear programs[J]. Operations Research Letters, 1999, 25(1): 1-13

[2] Black F,Litterman R.Global portfolio optimization[J].Financial Analysis Journal,1992,(48):28-43.

[3] D. Goldfaxb, G. Iyengar. Robust portfolio selection problems [J].Mathematics of Operations Research, 2003, 97: 138.

[4] Fabozzi J F,Klom P N,Pachamanova D A,Foeardi S M.Robust portfolio optimization[J].The Journal "Portfolio Management,2007.40.48.

[5] Gao Ying, Li Chajun, Tang Shiyuan. The application of portfolio model based on robust optimization in investment fund [J]. Journal of Northeastern University (natural science edition), 2009,24(2) : 295-297

[6] Huang Xiaoyuan, Gao Ying. Card network robust operation model in uncertain fund demand [J]. Control and decision, 2007, 22(10) 1147.1150

[7] Lu. Z. Robust portfolio selection based on a joint ellipsoidal uncertainty set [J]. Optimization Methods \& Software, 2011, 26(1): 89-104

[8] Mulvey J M, Vanderbei R J,Zenios S A.Robust optimization of large-scale systems[J].Operations Research, 1995, 43.(2):264-281.

[9] Wang Yuanying, Ye Ahongxing. Undetermined optimal investment portfolio in market conditions [J]. Journal of operational research, 2007, 11(4): 102-108.

[10] William t. zimba, John m. marvey. Global asset and liability management modeling [M]. Gu Juan et al. Beijing: economic science press, 2003, 55. 64.

[11] Zhu Shushang, Li Rui, Zhou Xunyu, Wang Shouyang. The theory of investment portfolio and financial optimization [J]. Journal of management science, 2004, 7(6): 1.10. 